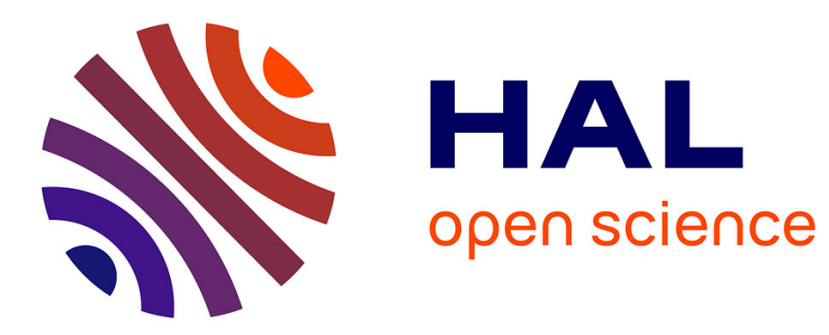

\title{
Optical flow with structure information for epithelial image mosaicing
}

Sharib Ali, Khuram Faraz, Christian Daul, Walter C.P.M. Blondel

\section{To cite this version:}

Sharib Ali, Khuram Faraz, Christian Daul, Walter C.P.M. Blondel. Optical flow with structure information for epithelial image mosaicing. 37th Annual International Conference of the IEEE Engineering in Medicine and Biology Society, EMBC 2015, Aug 2015, Milano, Italy. hal-01184618

\section{HAL Id: hal-01184618 \\ https://hal.science/hal-01184618}

Submitted on 5 Nov 2015

HAL is a multi-disciplinary open access archive for the deposit and dissemination of scientific research documents, whether they are published or not. The documents may come from teaching and research institutions in France or abroad, or from public or private research centers.
L'archive ouverte pluridisciplinaire HAL, est destinée au dépôt et à la diffusion de documents scientifiques de niveau recherche, publiés ou non, émanant des établissements d'enseignement et de recherche français ou étrangers, des laboratoires publics ou privés. 


\title{
Optical flow with structure information for epithelial image mosaicing
}

\author{
Sharib Ali ${ }^{1,2}$, Khuram Faraz ${ }^{1,2}$, Christian Daul ${ }^{1,2}$ and Walter Blondel ${ }^{1,2}$
}

\begin{abstract}
Mosaicing of biological tissue surfaces is challenging due to the weak image textures. This contribution presents a mosaicing algorithm based on a robust and accurate variational optical flow scheme. A Riesz pyramid based multiscale approach aims at overcoming the "flattening-out" problem at coarser levels. Moreover, the structure information present in images of epithelial surfaces is incorporated into the data-term to improve the algorithm robustness. The algorithm accuracy is first assessed with simulated sequences and then used for mosaicing standard clinical endoscopic data.
\end{abstract}

\section{INTRODUCTION}

Video-sequences of biological tissue surfaces are acquired in vivo in various medical fields where visual inspection is needed such as dermatology [1], urology [2], [3] and gastroscopy [4]. However, the small field of view (FOV) of these video-images does not facilitate lesion diagnosis, patient follow-up and data archiving. Such limitations can be overcome by building large FOV mosaics. One crucial step in image mosaicing is the determination of the correspondence between homologous pixels of image pairs. This correspondence allows for computing the geometrical transformations between image pairs required to place all frames in a global mosaic coordinate system. The method to be used to establish the correspondence between homologous pixels depend on the image content and quality.

Images of tissue surfaces acquired with optical sensors are affected by non-constant contrast due to varying imaging conditions (e.g. illumination changes between images or blur) and changing scene characteristics (e.g. high inter- and intra-patient texture variability). Although "feature based" approaches can be appropriate when the textures are constrasted (for instance the method in [5] used in fluorescence cystoscopy), they are unable to establish unambiguous correspondences in complicated scenes with weakly pronounced textures. "Pixel based" methods are more suitable to deal with large texture variability and non-uniform illumination conditions. Such methods have been successfully used in bladder image mosaicing. A mutual information based similarity measure was maximized using a stochastic gradient in [6] and the sum of squared differences was minimized using a graph-cut based optimization in [2] for robust image mosaicing of cystoscopic images. However, these methods are very slow and not convenient for preoperative diagnosis. Recently for cystoscopic images, the best compromise between registration robustness, accuracy and speed has been achieved using optical flow (OF) techniques [3], [7].

\footnotetext{
${ }^{1}$ Université de Lorraine, CRAN, UMR 7039, 2 avenue de la Forêt de Haye, 54516 Vandœuvre-lès-Nancy, France

${ }^{2}$ CNRS, CRAN, UMR 7039, 54516 Vandœuvre-lès-Nancy, France
}

The choice of an OF scheme for a given application depends on the iconic information and noise present in the scene and other physical conditions like illumination variations, surface reflectance etc. Thus, in epithelium images, local OF methods give inaccurate flow vectors in image regions with low texture [8], [7], while global variational approaches with an $l^{2}$ data fidelity term and quadratic regularizer [9] lead to outliers and oversmoothed flow fields. Independently of a scene type, these problems were addressed by using $l^{1}$ dataterms and regularizer allowing for piecewise-smooth flow field [10], [11]. Classically, brightness constancy assumption (BCA) is used in the data-term by numerous algorithms. This is an inappropriate assumption for most of the epithelium video-images affected by a large variability in illumination, often due to viewpoint changes. Moreover, when epithelium is acquired with endoscopes, additional vignetting effect also affects the images. To allow some small variations in grey value, a gradient constancy assumption (GCA) was used in [10] as a complementary information in the data term. However, this assumption cannot handle large in-plane rotations which usually occur in epithelial video-sequences like in cystoscopy or dermatology. It was shown in [3], [12] that adding structure constancy assumption (SCA) leads to very robust data term for epithelial image mosaicing. Although epithelial regions generally do not possess salient features, some less prominent structures can be exploited using structure enhancement techniques.

Variational OF methods use coarse-to-fine energy minimization approaches to handle large displacements. However, using original images in a multi-resolution approach leads to the "flattening-out" problem in image regions at coarser levels. This results in poor initialization of the OF field from coarser to finer levels. Wavelet based methods can deal with this problem and are directly integrable in a variational energy formulation due to their inbuilt coarseto-fine property. Such approach is used in [13] to overcome both the flattening-out problem and the response supression due to point-noise. Wavelet coefficients are used to compute the OF instead of using directly the grey-level pixel values. However, the major limitation is that local OF approaches give inaccurate flow field for low texture images.

In this paper, we present a wavelet based multi-resolution approach for accurate and robust OF computation in a variational framework. We make use of structures existing in epithelial tissue images to model a novel data-term in this scheme. Section 2 of this contribution details the proposed algorithm. In section 3, we provide both quantitative and qualitative results on images of different organ surfaces. Finally, we conclude the paper in section 4 . 


\section{OF ALGORITHM FOR EPITHELIAL TISSUE IMAGES}

\section{A. Variational approach}

Let $I_{i}$ and $I_{i+1}$ be the target and the source images of a video-sequence. The energy to be minimized for $\mathrm{OF}$ estimation consists of a data-term and a smoothness term [9]. Let $\mathbf{x}=\{x, y\} \in \mathbb{R}^{2}$ be the pixel coordinates in space $\Omega$ of images $\left\{I_{i}(\mathbf{x}), I_{i+1}(\mathbf{x}+\mathbf{u})\right\}$ with $\mathbf{u}=\{u, v\}: \Omega \rightarrow \mathbb{R}^{2}$ being the OF field with vector components $u$ and $v$ along the $x-$ and $y$ - image axes respectively. Then, the minimization of energy $E(\mathbf{u})$ can be represented as the weighted sum of a data term and a regularization term as:

$$
E(\mathbf{u})=E_{\text {data }}(\mathbf{u})+\lambda E_{\text {reg }}(\mathbf{u}),
$$

where $E_{\text {data }}$ is the data-term established between images $I_{i}(\mathbf{x})$ and $I_{i+1}(\mathbf{x}+\mathbf{u}), E_{r e g}$ stands for the regularizer making the estimation of OF field $\mathbf{u}$ piecewise smooth and $\lambda$ is the tradeoff between both terms of $E(\mathbf{u})$.

\section{B. Riesz pyramid and structure estimates}

A coarse-to-fine strategy has been used to handle large displacements. However, for accurate OF initialization at coarse levels, it is important to preserve structure information by attenuating the flattening-out effects. To do so, a combination of two approaches preserving image structure at coarse levels are presented in this section.

1) Wavelet based coarse-to-fine approach: Mallat's multiresolution analysis of $L_{2}\left(\mathbb{R}^{2}\right)$ [14] has been used for decomposition of images into wavelet sub-spaces. The choice of appropriate wavelet is crucial for structure preservation and attenuation of high frequency noise. Less pronounced edges and structures are usually not preserved by separable orthogonal wavelet decomposition, mainly because the corresponding filters are not rotation invariant. However, Riesz basis filters have an arbitrary steering property with appropriate linear combination of its set of directional filters [15]. We use a $2^{\text {nd }}$-order Riesz transform to decompose the image pairs $\left(I_{i}(\mathbf{x}), I_{i+1}(\mathbf{x}+\mathbf{u})\right)$ into $j$ scales.

For a wavelet subspace $V_{j}$, the decomposition of images $\left\{I_{i}, I_{i+1}\right\} \in \mathbb{R}^{2}$ at scale $j$ can be represented as the projection of the images onto $V_{j}$ with the Riesz basis filters $R^{N}$ :

$$
\begin{array}{r}
R I_{i}^{j}(\mathbf{x})=\operatorname{proj} \cdot V_{j} I_{i}=\sum_{k \in \mathbb{Z}^{2}}\left\langle R^{N} \varphi_{j, k}, I_{i}(\mathbf{x}) * G_{j}\right\rangle R \hat{\varphi}_{j, k} \\
R I_{i+1}^{j}(\mathbf{x}+\mathbf{u})=\operatorname{proj} \cdot V_{j} I_{i+1}=\sum_{k \in \mathbb{Z}^{2}}\left\langle R^{N} \varphi_{j, k}, I_{i+1}(\mathbf{x}+\mathbf{u}) * G_{j}\right\rangle R \hat{\varphi}_{j, k},
\end{array}
$$

where $G_{j}$ is the Difference of Gaussians (DoG) kernel at scale $j$ and shift $k$. A linear combination of an adaptive directional image is thus obtained using such filter-banks for a given scale $j$. A DoG kernel has been used in order to minimize the loss of details in the coarser resolutions while attenuating the high frequency noise. The Riesz basis $R^{N} \varphi_{j, k}$ is given by:

$$
R^{N} \varphi_{j, k}=2^{-j} R^{N} \varphi_{0, k}\left(2^{-j} \mathbf{x}-k\right),
$$

with shift $k=\left(k_{x}, k_{y}\right)$ along $x-$ and $y-$ axes. $R^{N} \hat{\varphi}_{j, k}$ is the dual basis of $R^{N} \varphi_{j, k}$.
2) Structure estimate on Riesz wavelet scales: The directional Riesz basis filters at each scale $j$ enhances the edges of structures, like blood vessels, lesions or pores of epithelium. As a result, it is possible to extract both the orientation and shape information of these structures even in low resolution image pairs. For estimating image structures, the Hessian matrix $H_{2} I_{i+1}^{j}(\mathbf{x}+\mathbf{u})$ of image $R I_{i+1}^{j}\left(\mathbf{x}+\mathbf{u}^{j}\right)$ is computed at each pixel $\mathbf{x}$ as in Eq. (4).

$$
H_{2} I_{i+1}^{j}(\mathbf{x}+\mathbf{u})=\left(\begin{array}{ll}
\frac{\partial^{2} R I_{i+1}^{j}\left(\mathbf{x}+\mathbf{u}^{j}\right)}{\partial x^{2}} & \frac{\partial^{2} R I_{i+1}^{j}\left(\mathbf{x}+\mathbf{u}^{j}\right)}{\partial x \partial y} \\
\frac{\partial^{2} R I_{i+1}^{j}\left(\mathbf{x}+\mathbf{u}^{j}\right)}{\partial x \partial y} & \frac{\partial^{2} R I_{i+1}^{j}\left(\mathbf{x}+\mathbf{u}^{j}\right)}{\partial y^{2}}
\end{array}\right)
$$

The eigenvalues $\lambda_{1}$ and $\lambda_{2}$ of $H_{2} I_{i+1}^{j}(\mathbf{x}+\mathbf{u})$ are then used for a structure estimate $S_{i+1}^{j}(\mathbf{x}+\mathbf{u})[3]$ :

$$
S_{i+l}^{j}(\mathbf{x}+\mathbf{u})=e^{\left(-R_{b}^{2} / 2 \beta_{1}^{2}\right)}\left(1-e^{\left(-R_{s}^{2} / 2 \beta_{2}^{2}\right)}\right),
$$

where $R_{b}=\lambda_{1} / \lambda_{2} \mid \lambda_{2} \geq \lambda_{1}$ is the blob measure of an object structure in the image, $R_{s}=\sqrt{\lambda_{1}^{2}+\lambda_{2}^{2}}$ represents the presence of object structures and $\left\{\beta_{1}, \beta_{2}\right\}$ are sensitivity control parameters empirically set to 0.5 each. $R_{b}$ relates to the eccentricity of the ellipse approximating an object shape (the eccentricity is large for structures like vessels or skin pores). $R_{s}$ is ideally zero when no structure is present.

\section{Energy function modeling}

Classically, only brightness constancy assumption (BCA) is used in the data term for energy minimization of $E(\mathbf{u})$ in Eq. (1). But, BCA alone on Riesz wavelet scale-space is not sufficient for minimizing $E(\mathbf{u})$ since Reisz filters do not preserve the original grey-level values. For this reason, $\mathrm{BCA}$ is complemented with a gradient constancy assumption (GCA, [10]) which is useful when there is directional change in intensity. Structure constancy assumption (SCA, [3]) has been integrated as an another data term. This is motivated by the fact that SCA relates to the structure enhancement by Riesz filters. Such image structures increase the robustness of OF estimation. Integrating BCA, GCA and SCA leads to a well posed data term at scale $j$ of a Riesz pyramid:

$$
\begin{aligned}
E_{\text {data }}\left(\mathbf{u}^{j}\right)= & \int_{\Omega} \psi\left(\left|R I_{i+1}^{j}\left(\mathbf{x}+\mathbf{u}^{j}\right)-R I_{i}^{j}(\mathbf{x})\right|^{2}+\right. \\
& \gamma_{1}\left|\nabla R I_{i+1}^{j}\left(\mathbf{x}+\mathbf{u}^{j}\right)-\nabla R I_{i}^{j}(\mathbf{x})\right|^{2}+, \\
& \left.\gamma_{2}\left|S_{i+1}^{j}\left(\mathbf{x}+\mathbf{u}^{j}\right)-S_{i}^{j}(\mathbf{x})\right|^{2}\right) d \mathbf{x}
\end{aligned},
$$

where $\gamma_{1}$ and $\gamma_{2}$ are the weighting parameters for the GCA and the SCA and $S_{i}^{j}$ and $S_{i+1}^{j}$ are structure estimates of images $R I_{i}^{j}$ and $R I_{i+1}^{j}$ respectively at scale $j$.

To deal with the aperture problem in OF estimate and outliers in the data term, regularizer has to be used in the minimization of $E(\mathbf{u})$. A classical regularizer in Eq. (7) preserves the motion discontinuities along the object boundaries. This leads to a piecewise smooth flow field.

$$
E_{r e g}\left(\mathbf{u}^{j}\right)=\phi\left(\left|\nabla u^{j}\right|^{2}+\left|\nabla v^{j}\right|^{2}\right) .
$$




\begin{tabular}{|c|c|c|c|c|c|}
\hline Dataset & $\theta$ & $s_{x}, s_{y}$ & $f_{x}, f_{y}$ & $h_{1}, h_{2}$ & $\sqrt{t_{x}^{2}+t_{y}^{2}}$ \\
\hline Data-I & $\pm 10^{0}$ & $0.90-1.10$ & $0.90-1.10$ & $\pm 10^{-5}$ & 70 \\
\hline Data-II & $\pm 5^{0}$ & $0.95-1.05$ & $0.95-1.05$ & $\pm 10^{-5}$ & 50 \\
\hline
\end{tabular}

TABLE I: $H_{i, i+1}^{\text {true }}$ homography parameter intervals used for computing the displacements between consecutive images. $\theta,\left\{s_{x}, s_{y}\right\}$, $\left\{f_{x}, f_{y}\right\},\left\{t_{x}, t_{y}\right\}$ and $\left\{h_{1}, h_{2}\right\}$ are the in-plane rotation, shear, scale, translation and perspective parameters respectively.

\section{Optimization}

$\psi($.$) and \phi($.$) are the robust functions represented as$ the data term and the regularizer in Eq. (6) and Eq. (7) respectively. We restrict these functions to be $l^{1}$-norm enforcing piecewise smooth flow field. So, $\psi(x)$ and $\phi(x)$ can be expressed as $\sqrt{x^{2}+\varepsilon^{2}}$ with very small $\varepsilon$. Such objective function is highly non-convex, however a coarseto-fine refining scheme with image warping strategy can avoid inaccuracies in flow field. We have used the numerical methods similar to Brox et al. [10].

\section{E. Image mosaicing}

Consecutive image pairs $\left(I_{i}\right.$ and $\left.I_{i+1}\right)$ of a given videoimage sequence are registered by computing their local homographies $H_{i, i+1}^{e s t}$ between them. These homographies are estimated with the correspondence between the homologous pixels given by the flow field vectors as in [7]. All these images are then stitched in a global coordinate frame by concatenating their local homographies as in Eq. (8).

$$
H_{0, i}^{e s t}=\prod_{k=0}^{k=i-1} H_{i-k-1, i-k}^{e s t}
$$

$H_{0, i}^{e s t}$ is the global homography placing the pixels of images $I_{i}$ in the mosaic coordinate system given by $I_{0}$ (first image of the sequence).

\section{RESULTS AND DISCUSSION}

\section{A. Dataset}

High resolution images of 1) a human skin surface and 2) an excised pig bladder inner surface (texture similar to human bladder) were first acquired. A dataset with 50 images each (data-I for skin and data-II for pig bladder) were extracted. A subimage $I_{0}$ was first chosen to define a reference coordinate system in the high resolution images. Then, the images $I_{1}$ to $I_{49}$ were extracted with known global transformation $H_{0, i}^{\text {true }}$, computed using simulated local transformations $H_{i, i+1}^{\text {true }}$ superimposing exactly image $I_{i+1}$ on $I_{i}$. General characteristics of these homographies are shown in Table I.

\begin{tabular}{|c|c|c|c|c|c|}
\hline \multirow{2}{*}{ Method } & \multicolumn{3}{|c|}{$\varepsilon_{i, i+1}^{\text {local (in pixel) }}$} & \multirow{2}{*}{$\begin{array}{c}\varepsilon_{0,49}^{\text {global }} \\
\text { (in pixel) }\end{array}$} & \multirow{2}{*}{$\begin{array}{c}\overline{\mathbf{t}} \\
(\text { in } \mathrm{s})\end{array}$} \\
\hline & $\min$ & $\max$ & mean & & \\
\hline HAOF [10] & 0.50 & 6.87 & 2.59 & 45 & 9 \\
\hline Graph-cut method [2] & 0.18 & 4.72 & 0.84 & 36 & 20 \\
\hline RFlow method [3] & 0.15 & 2.23 & 0.70 & 30 & 4 \\
\hline Proposed method & 0.04 & 1.32 & 0.37 & 9.1 & 5 \\
\hline
\end{tabular}

TABLE II: Method comparison on data-I (human skin epithelium).

\begin{tabular}{|l|l|l|l|c|c|}
\hline \multirow{2}{*}{ Method } & \multicolumn{3}{|c|}{$\varepsilon_{i, i+1}^{\text {local }}$ (in pixel) } & \multirow{2}{\varepsilon_{0,49}^{\text{global}}}{} & $\begin{array}{c}\overline{\mathbf{t}} \\
\text { (in pixel) }\end{array}$ \\
\cline { 2 - 4 } & min s) & max & mean & 17 & 13 \\
\hline HAOF [10] & 0.66 & 4.87 & 3.77 & 11 & 48 \\
\hline Graph-cut method [2] & 0.32 & 6.8 & 3.01 & 11 & $\mathbf{3}$ \\
\hline RFlow method [3] & $\mathbf{0 . 0 2}$ & 1.56 & 0.33 & 7.5 & $\mathbf{3}$ \\
\hline Proposed method & 0.03 & $\mathbf{1 . 0 6}$ & $\mathbf{0 . 2 1}$ & $\mathbf{4 . 4}$ & 5 \\
\hline
\end{tabular}

TABLE III: Method comparison on data-II (pig bladder).

\section{B. Quantitative evaluation for epithelium tissue registration}

Two criteria (see Eq. (9)) were used for comparing the proposed method with some recent methods in bladder epithelium mosaicing. The local error $\varepsilon_{i, i+1}^{\text {local }}$ gives the registration accuracy when superimposing pixels $p$ of images $I_{i}$ and $I_{i+1}$, whereas $\varepsilon_{0,49}^{\text {global }}$ is the global (mosaicing) error when placing image $I_{49}$ in the coordinate system of $I_{0}$.

$$
\begin{aligned}
& \varepsilon_{i, i+1}^{\text {local }}=\frac{1}{N} \sum_{p \in I_{i} \cap I_{i+1}}\left\|H_{i, i+1}^{\text {true }} p-H_{i, i+1}^{\text {est }} p\right\|^{2} \\
& \varepsilon_{0,49}^{\text {global }}=\frac{1}{N} \sum_{p \in I_{0} \cap I_{49}}\left\|H_{0,49}^{\text {true }} p-H_{0,49}^{\text {est }} p\right\|^{2} .
\end{aligned}
$$

Table II and Table III give the results for the human skin epithelium (data-I) and the pig bladder epithelium (data-II) respectively. In comparison to all the reference methods, the proposed method has the least of the maximum local registration error for both datasets. The HAOF method ([10]) and the graph-cut based method (GC, [2]) recorded large maximal local registration errors of 6.87 and 6.8 pixels for skin and bladder epithelium respectively. Moreover, the proposed method has the lowest average local registration error for both the datasets. For the skin epithelium sequence, the error of all the reference methods is atleast twice larger than that of the proposed method. For the bladder data, only RFLOW has an accuracy (average $\varepsilon_{i, i+1}=0.33$ pixels) comparable to that of the proposed method (average $\varepsilon_{i, i+1}=$ 0.21 pixels). The proposed method has also the least global registration error with 9.1 pixels for skin data and 4.4 pixels for pig bladder epithelium. This led to coherent mosaics given in Figs. 1 and 2. The proposed method has also comparable average computational time ( $\bar{t}$ in Tables II and III) with the convex RFLOW model but with much higher registration accuracy.

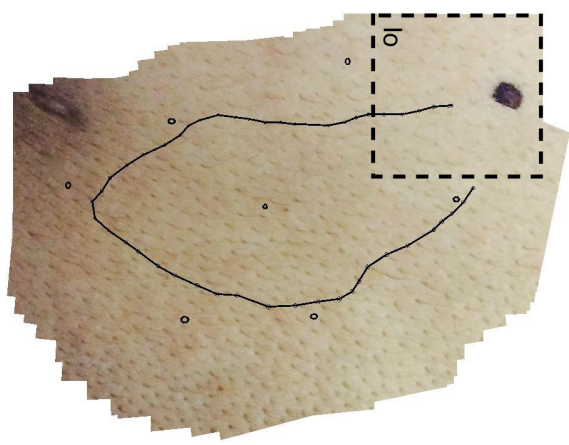

Fig. 1: Human skin data-I mosaic with the proposed method. $900 \times$ 1400 pixels mosaic was obtained with $I_{0}$ being the first image. 


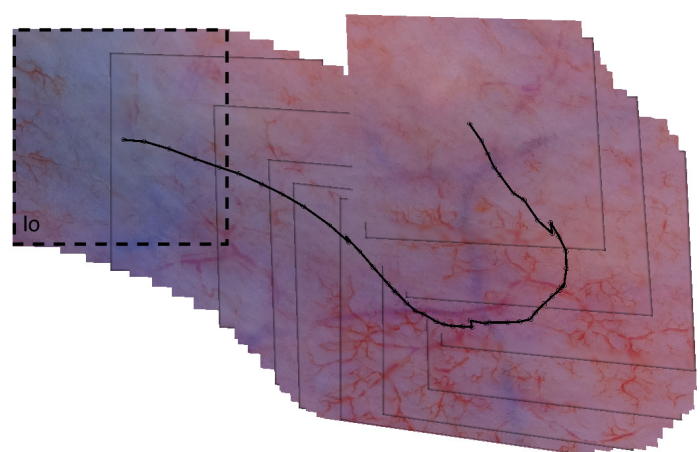

Fig. 2: Mosaic of data-II (pig bladder) with estimated $H_{i, i+1}^{e s t}$ using the proposed method. A mosaic of $900 \times 1500$ pixels was obtained.

\section{Qualitative evaluation on clinical data}

Complementary qualitative validation of robustness of the proposed method against variability of epithelial textures has also been done on cystoscopic patient data and gastroscopic patient data acquired during standard procedures at the Institut de Cancérologie de Lorraine of Nancy and at the Ambroise Paré hospital of Paris respectively after the concent from their ethical committees. Fig. 3 shows a coherent mosaic (i.e. without texture discontinuities) of a human inner-bladder epithelium. Fig. 4 gives mosaic of a stomach part (pyloric antrum region) acquired with a gastroscope.

\section{CONCLUSION}

In this paper, structures of epithelial surfaces have been exploited for robust and accurate OF estimation for establishing pixel correspondence between image pairs and leading to coherent mosaicing of these surfaces. Further work will be to perform extended tests of the proposed approach on epithelial images acquired under other imaging protocols like confocal microscopy and fluorescence imaging.

\section{ACKNOWLEDGEMENT}

The $\mathrm{PhD}$ grant of $\mathrm{S}$. Ali is co-funded by the Agence nationale de la recherche and the Conseil régional de Lorraine in the framework of CyPaM2 ANR-11-TECS-001 project. The $\mathrm{PhD}$

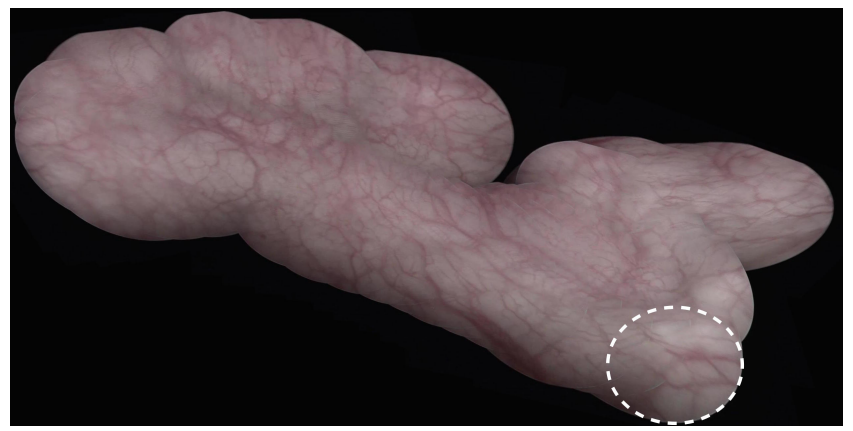

Fig. 3: Human inner bladder wall mosaic using the proposed method. It uses 900 frames corresponding to $35 \mathrm{~s}$ of cystoscopic video data. The white circle represents the FOV of a video frame.

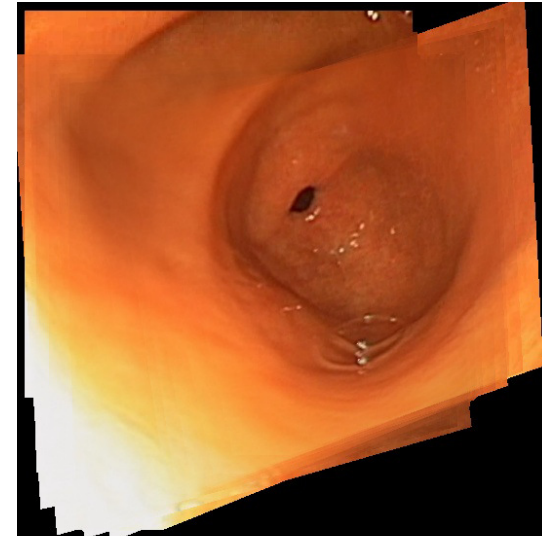

Fig. 4: Mosaic for pyloric antrum region using 50 image pairs (gastroscopy). Air bubble and specular reflections are observable.

grant of K. Faraz is co-funded by the European Regional Development Fund and the Conseil régional de Lorraine in the framework of the InnovaTICs-Dépendance project.

\section{REFERENCES}

[1] K. Loewke, D. Camarillo, W. Piyawattanametha, M. Mandella, C. Contag, S. Thrun, and J. Salisbury, "In vivo micro-image mosaicing," IEEE Trans. on Biomed. Eng., vol. 58, pp. 159-171, 2011.

[2] T. Weibel, C. Daul, D. Wolf, R. Rösch, and F. Guillemin, "Graph based construction of textured large field of view mosaics for bladder cancer diagnosis," Pat. Recog., vol. 45, pp. 4138-4150, 2012.

[3] S. Ali, C. Daul, and W. Blondel, "Robust and accurate optical flow estimation for weak texture and varying illumination condition: Application to cystoscopy," in 4th Int. Conf. on Img. Proc. Theory, Tools and Appl. (IPTA), 2014, pp. 1-6.

[4] S. Seshamani, W. Lau, and G. Hager, "Real-time endoscopic mosaicking," in Med. Img. Comp. and Comp.-Assist. Interv. (MICCAI)

[5] A. Behrens, T. Stehle, S. Gross, and T. Aach, "Local and global panoramic imaging for fluorescence bladder endoscopy," in Intl. Conf. of the IEEE Eng. in Med. and Bio. Soc. (EMBC), 2009, pp. 6690-93.

[6] R. Miranda-Luna, C. Daul, W. Blondel, Y. Hernandez-Mier, D. Wolf, and F. Guillemin, "Mosaicing of bladder endoscopic image sequences: Distortion calibration and registration algorithm," IEEE Trans. Biomed. Engineering, vol. 55, no. 2, pp. 541-553, 2008.

[7] S. Ali, C. Daul, T. Weibel, and W. Blondel, "Fast mosaicing of cystoscopic images from dense correspondence: combined surf and TV-L1 optical flow method," in IEEE Int. Conf. on Image Processing (ICIP), 2013, pp. 1291-1295.

[8] Y. Hernandez-Mier, W. Blondel, C. Daul, D. Wolf, and F. Guillemin, "Fast construction of panoramic images for cystoscopic exploration," Comp. Med. Imag. and Graph., vol. 34, no. 7, pp. 579-592, 2010.

[9] B. Horn and B. Schunck, "Determining optical flow," Artificial Intelligence, vol. 17, pp. 185-203, 1981.

[10] T. Brox, A. Bruhn, N. Papenberg, and J. Weickert, "High accuracy optical flow estimation based on a theory for warping," in Proc. Euro. Conf. Comp. Vis. (ECCV), 2004, vol. 4, pp. 25-36.

[11] C. Zach, T. Pock, and H. Bischof, "A duality based approach for realtime TV-L1 optical flow," in Ann. Symp. German Association Patt. Recogn, 2007, pp. 214-223.

[12] S. Ali, C. Daul, E. Galbrun, M. Amouroux, F. Guillemin, and W. Blondel, "Robust bladder image registration by redefining dataterm in total variational approach," in Proc. SPIE Medical imaging, Image Processing conference, 2015, vol. 9413, pp. 1H-12.

[13] H. Liu, R. Chellappa, and A. Rosenfeld, "Fast two-frame multiscale dense optical flow estimation using discrete wavelet filters," $J$. of the Opt. Soc. of Amer., vol. 20, pp. 1505 - 1515, 2003.

[14] S. G. Mallat, "A theory for multiresolution signal decomposition: the wavelet representation," IEEE Trans. on Patt. Analy. and Mach. Intell., vol. 11, pp. 674-693, 1989.

[15] M. Unser, N. Chenouard, and D. Van De Ville, "Steerable pyramids and tight wavelet frames in $L_{2}\left(\mathrm{R}^{d}\right) .$, IEEE Transactions on Image Processing, vol. 20, no. 10, pp. 2705-2721, 2011. 